\title{
The recent results in the analysis of the meson production reactions
}

\author{
A. V. Sarantsev ${ }^{1,2}$ \\ ${ }^{1}$ HISKP, Bonn University, Bonn, Germany \\ ${ }^{2}$ PNPI, NRC Kurchatov Institute, Gatchina, Russia
}

Abstract. The recent results from the Bonn-Gatchina group are presented and discussed. The analysis of the new data on the $\eta$ and $\eta^{\prime}$ photoproduction off proton firmly confirms the existence of three nucleon resonances with masses around $1900 \mathrm{MeV}$. The combined analysis of the data on the kaon proton collision reactions indicates the presence of new states which were missed in the analysis of the separate data sets.

\section{Introduction}

The precise information about spectrum of hadrons and their properties is the key point for understanding the strong interactions at low and intermediate energies. In the sector of light baryons the bound systems of three valence quarks should form multiplets: octets which consist of nucleons, $\Lambda, \Sigma$ and $\Xi$ states and decuplets which consist of the $\Delta, \Sigma, \Xi$ and $\Omega$ particles. In the case of more complicated systems with five or more valence quarks, baryons should form a more complicated multiplets.

If a state can not be classified as member of a quark multiplet it should be regarded as an exotic one from the point of view of the quark classification. Therefore it is very important to define all members of the multiplet. For such classification it is necessary to know branching ratios of the baryon states into different channels: such knowledge provides an important information about internal structure of states.

However the number of theoretically predicted states based on the quark classification is notably larger than the number of states observed experimentally. Indeed the number of the observed $\Sigma$-hyperons is less than the number of known nucleons and $\Delta$-states. For example, only one $1 / 2^{-} \Sigma$-hyperon was observed in the mass region below $1.9 \mathrm{GeV}$, while one expects three: the multiplet partners of $N(1535) 1 / 2^{-}, N(1620) 1 / 2^{-}$and $\Delta(1620) 1 / 2^{-}$.

Another problem concerns understanding the quark interaction forces. The spectrum of the nucleon and delta states predicted by the classical quark model [1] is not confirmed experimentally, due to missing a large number of excited states. It is very important to understand whether these missing states are members of specific multiplets. This will help to understand whether we have a problem with understanding the baryon structure or with our understanding the quark interaction forces or with missing experimental information.

Recently a number of the meson photo-production experiments were carried out over the world. The information obtained from the $\gamma$-nucleon collision reactions is complimentary to the information obtained from the pion-nucleon collision experiments and seriously improved our knowledge about baryon spectrum and properties of the observed states. 
For the hyperon resonance study the only data from the kaon-nucleon collision reactions are available for the analysis. Moreover until recently the partial wave analyses of such data were performed for the single experiments only which allowed a large flexibility of the solutions. Moreover, in this case one can not impose such basic properties as unitarity and analyticity conditions due to missing information about other decay channels. Recently a combined analysis of all available data on the kaon-nucleon collision was performed by the ANL-Osaca group [2]. The Bonn-Gatchina approach is based on the covariant energy dependent approach which allows us to perform the combined analysis of many reactions. It was developed in [3],[4] and was successfully applied to the analysis of the data on the meson photoproduction and pion-induced reactions (see, for example, [5]) and to the analysis of the data on the nucleon-nucleon collision reactions [6]. In this paper we present the result of our analysis of the latest meson photoproduction data and the combined analysis of the data on the kaon-proton collision reactions.

\section{The analysis of the new $\eta$ and $\eta^{\prime}$ photoproduction data}

The $\eta$ and $\eta^{\prime}$ photoproduction data provide a unique information about spectrum and properties of the nucleon states. Recently the CB-ELSA collaboration measured with a good precision $\gamma p \rightarrow \eta p$ differential cross section up to $2.3 \mathrm{GeV}$ and $\Sigma$ E,T,H,P,G observables while the A2 collaboration measured with a very high statistics the differential cross section up to $1.98 \mathrm{GeV}$ and T,F observables [7]. The combined analysis of these new data, together with the data from photo and pion induced reactions with other final states allowed us to extract the branching ratios of the contributing states to the $\eta p$ channel. Although the most of the found values are in a good agreement with PDG averages, we found a rather large branching ratio from the $N(1650) 1 / 2^{-}$state: $32 \pm 4 \%$. This number was calculated from the couplings extracted as residues at the pole position and due to a strong interference between $N(1535) 1 / 2^{-}, N(1650) 1 / 2^{-}$and $N(1895) 1 / 2^{-}$can be different from the fit were such interference is neglected. Another reason is that this ratio is defined by the data from the polarization observables which were not measured earlier.

The description of the A2 differential cross section and the contribution of the leading observables are shown in Fig. 1. The total cross section shows a sharp drop in the mass region $1900 \mathrm{MeV}$ which corresponds to the opening of the $\eta^{\prime} p$ threshold and the presence of the state $N(1895) 1 / 2^{-}$. This state has a rather strong coupling to the $\eta^{\prime}$-nucleon channel which leads to the drop of its contribution to the cross section.

In turn, the big $\eta^{\prime}$-nucleon coupling of $N(1895) 1 / 2^{-}$should be seen in the $\gamma p \rightarrow \eta^{\prime} p$ data. Therefore we included the corresponding differential cross section and the beam asymmetry data measured by the CLAS collaboration [8] in our combined analysis . The description of the $\gamma p \rightarrow \eta^{\prime} p$ differential cross section and the contribution of the leading partial waves to the total cross section are shown in Fig. 2. Indeed, $N(1895) 1 / 2^{-}$produces the main contribution near $\eta^{\prime} p$ threshold.

The mass and widths of the $N(1895) 1 / 2^{-}$state was found to be in a good agreement with the results of the previous analysis: $M=1895 \pm 15 \mathrm{MeV}$ and width $\Gamma=90_{-15}^{+30}$. This result was confirmed by the independent analysis of the same data sets performed by the MAID group which found the mass of the state $M=1896 \pm 1$ and $\Gamma=93 \pm 13 \mathrm{MeV}$ [7].

\section{Analysis of the $\gamma n \rightarrow K \Lambda, K \Sigma$ data}

The new data on the kaon photoproduction off neutron were measured by the CLAS collaboration [9],[10]. These data together with the $\gamma n \rightarrow \pi^{-} p, \gamma n \rightarrow \pi^{0} n$ and $\gamma n \rightarrow \eta n$ data 

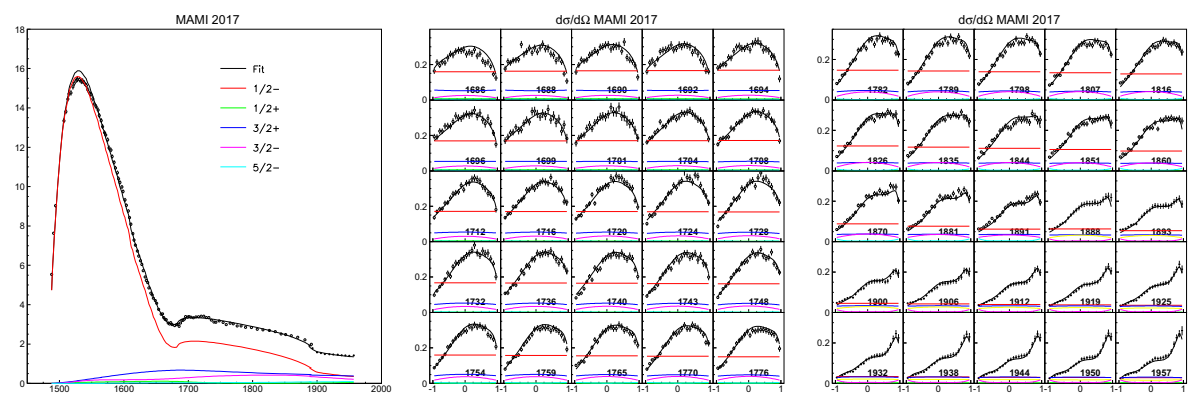

Figure 1. Description of the $\gamma p \rightarrow \eta p$ differential cross section from A2 collaboration [7] and contribution of the leading partial waves.
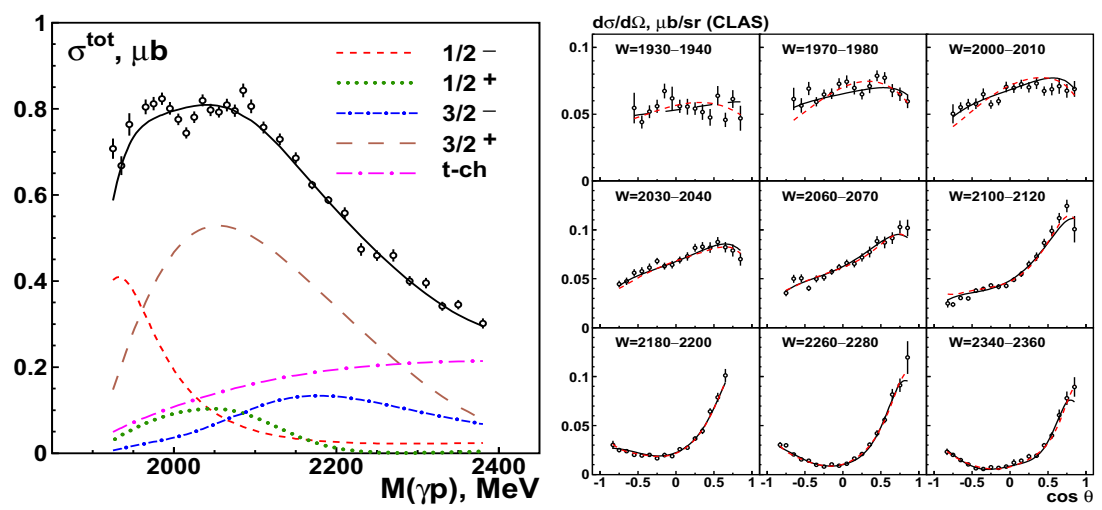

Figure 2. Description of the $\gamma p \rightarrow \eta^{\prime} p$ differential cross section from CLAS [8] and the contribution of the leading partial waves to the total cross section.

provide a strong constrain on the $\gamma$-neutron couplings of the nucleon states. Apart from this, the new data show strong signals from the states which provided only some improvements in the description of the $\gamma p$ collision data and needed a conformation. The description of the $\gamma n \rightarrow K^{0} \Lambda$ differential cross section and contributions of the leading partial wave to the total one are shown in Fig. 3. The corresponding pictures for the $\gamma n \rightarrow K^{+} \Sigma^{-}$data are shown in Fig 4. Due to a lack of the data at forward and backward angles we have found the set of the solutions which describe equally well the fitted data. However in all solutions the dominant contributions are defined by the $N(1895) 1 / 2^{-}, N(1880) 1 / 2^{+}$and $N(1900) 3 / 2^{+}$states. All these states were observed earlier in the $\gamma p$ collision reactions and are firmly confirmed by the present data. However the mass of the $N(1880) 1 / 2^{+}$state was optimized at slightly higher value: $M=1950 \pm 25 \mathrm{MeV}$.

\section{Hyperon spectrum}

The identification of the new states in the meson photoproduction data should be accompanied with an identification of the corresponding nonet members in the hyperon spectrum. However even in the case of the well known nucleon and delta resonances the corresponding 

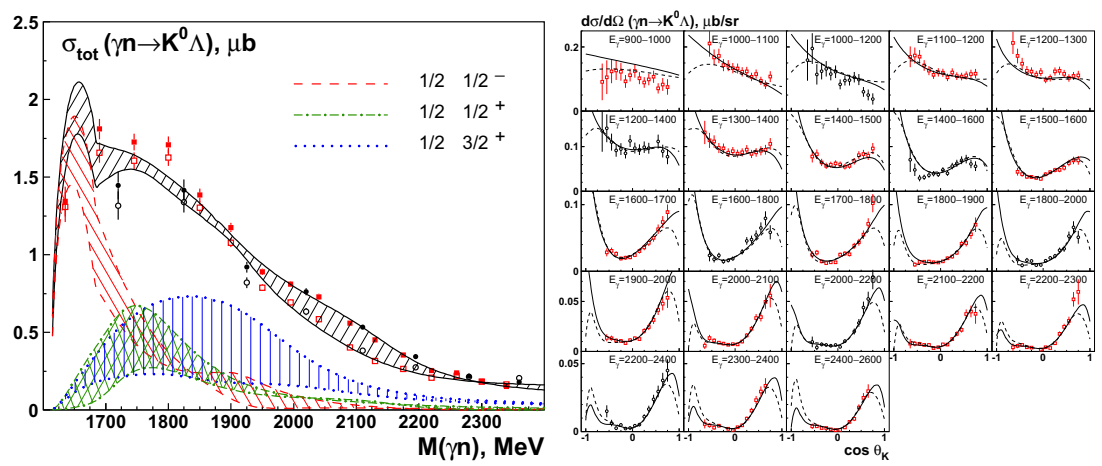

Figure 3. The contributions of the leading partial wave to the $\gamma n \rightarrow K^{0} \Lambda$ total cross section and the description of the differential cross section [9] with two found solutions.
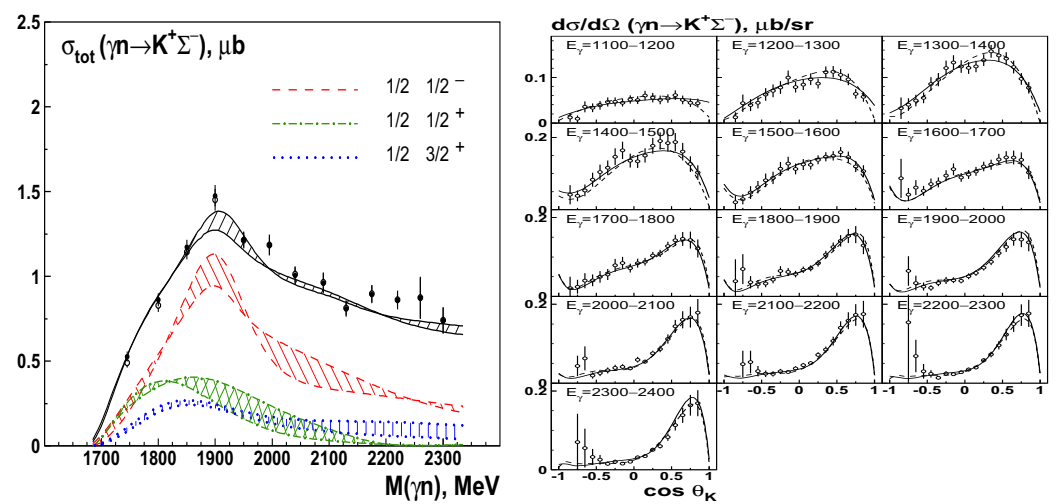

Figure 4. The description of the CLAS data on the $\gamma n \rightarrow K^{+} \Sigma^{-}$differential cross section [10].

hyperon states are not identified. To clarify this situation we have performed the combined analysis of the data on the kaon-proton collision. As the starting point we included in the fit all resonances with 3 and 4-star rating from PDG. Such fit showed a good description for the elastic channel but reveals systematical deviations in the mass region $1.65-1.90 \mathrm{GeV}$ for the description of the $K p \rightarrow \pi^{0} \Lambda, K p \rightarrow \pi^{+} \Sigma^{-}$and $K p \rightarrow \pi^{-} \Sigma^{+}$data. To study wether these deviations are due to missing states we have added in all partial waves one by one the $\Lambda$ and $\Sigma$ resonances and performed the mass scan of the introduced states. In the $\Lambda$ sector we observed only an improvement from the $3 / 2^{+}$state located in the mass region around $1900 \mathrm{MeV}$. In the $\Sigma$-hyperon sector we observe two rather promising minima in the $1 / 2^{-}$partial wave and one minimum above $2 \mathrm{GeV}$ in the $5 / 2^{-}, 7 / 2^{-}, 3 / 2^{+}$partial waves.

The new state in the $1 / 2^{-}$partial wave optimized with the mass $1670 \mathrm{MeV}$ and width $60 \mathrm{MeV}$. The mass and width are close to the well known state $\Sigma\left(3 / 2^{-}\right)$. Probably this can explain why this state was missed in the analyses of the separate reactions. The solution with included new states produced a very good description of the data and was stable against adding new states to the fit. The description of the $K^{-} p \rightarrow \pi^{0} \Lambda$ data in the region around $1700 \mathrm{MeV}$ and contributions from the leading partial waves to the total cross section are shown in Fig. 5. The resonances observed in the $\Sigma$-hyperon spectrum are listed in Table 4. In our fit we observe two new states with quantum numbers $1 / 2^{-}$and one state with $J^{P}=5 / 2^{-}$. 
However the last one is situated above $2200 \mathrm{MeV}$ and needs a confirmation which can be obtained from the analysis of the future data with production of $\Sigma$-hyperons.
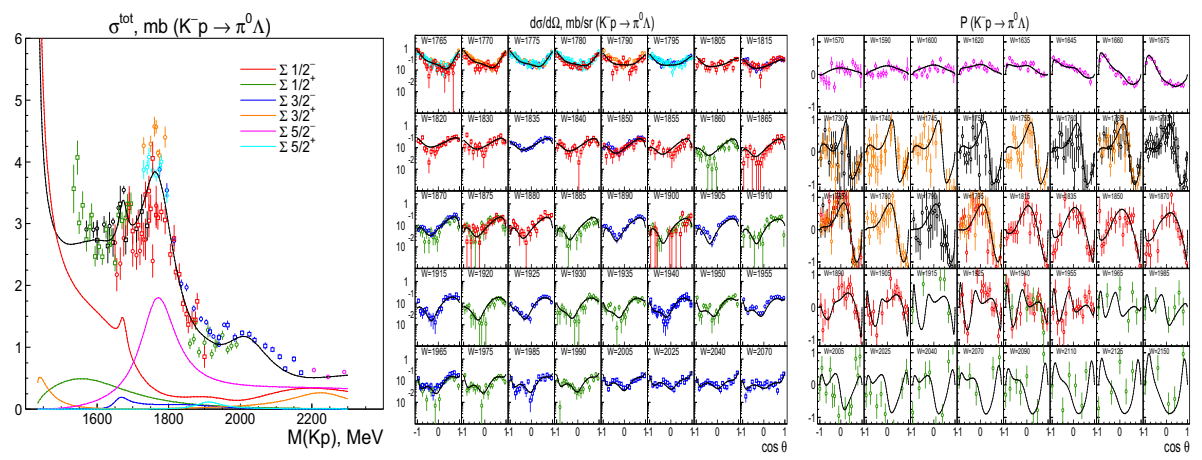

Figure 5. The description of the $K^{-} p \rightarrow K^{0} \Lambda$ data and the contributions from leading partial waves. The data are from [11], [12],[13], [14],[15], [16],[17], [18],[19],[20].

Table 1. $\Sigma$-Hyperons used in the first fit to the data.

\begin{tabular}{lllll}
\hline \hline & $J^{P}$ & Status & Mass & Width \\
\hline$\Sigma(1660)$ & $1 / 2^{+}$ & $* * *$ & $1630-1690$ & $36.0 \pm 0.7$ \\
$\Sigma(1385)$ & $3 / 2^{+}$ & $* * * *$ & $1382.80 \pm 0.35$ & $40-200$ \\
$\Sigma(1915)$ & $5 / 2^{+}$ & $* * * *$ & $1900-1935$ & $80-160$ \\
$\Sigma(2030)$ & $7 / 2^{+}$ & $* * * *$ & $2025-2040$ & $150-200$ \\
\hline$\Sigma(1670)$ & $3 / 2^{-}$ & $* * * *$ & $1665-1685$ & $40-80$ \\
$\Sigma(1750)$ & $1 / 2^{-}$ & $* * *$ & $1730-1800$ & $60-160$ \\
$\Sigma(1775)$ & $5 / 2^{-}$ & $* * * *$ & $1770-1780$ & $105-135$ \\
$\Sigma(1940)$ & $3 / 2^{-}$ & $* * *$ & $1900-1950$ & $150-300$ \\
\hline$\Sigma(1665)$ & $1 / 2^{-}$ & & $1670 \pm 15$ & $60 \pm 30$ \\
$\Sigma(2150)$ & $1 / 2^{-}$ & & $2160 \pm 20$ & $220 \pm 25$ \\
$\Sigma(2250)$ & $5 / 2^{-}$ & & $2250 \pm 30$ & $330 \pm 40$ \\
\hline
\end{tabular}

\section{Conclusion}

The new data on the $\eta$ and $\eta^{\prime}$ photoproduction off proton and new data on the kaon photoproduction off neutron strongly confirm the existence of three baryon resonances $N(1895) 1 / 2^{-}$, $N(1880) 1 / 2^{+}$and $N(1900) 3 / 2^{+}$. The new data allows us to define resonance branchings to the $\eta p$ channel and $\gamma n$ couplings of the observed states with a good accuracy.

The combined analysis of kaon-proton collision data reveals the presence of two new $\Sigma$ hyperons with quantum numbers $J^{P}=1 / 2^{-}$and a hint for the existence of the $\Sigma$ state with $J^{P}=5 / 2^{-}$in the mass region above $2.2 \mathrm{GeV}$.

This work was supported by the RSF grant 16-12-10267.

\section{References}

[1] S. Capstick and N. Isgur, Phys. Rev. D 34, 2809 (1986) 
[2] H. Kamano, S. X. Nakamura, T.-S. H. Lee and T. Sato, Phys. Rev. C 90, no. 6, 065204 (2014) doi:10.1103/PhysRevC.90.065204 [arXiv:1407.6839 [nucl-th]]

[3] A. Anisovich, E. Klempt, A. Sarantsev and U. Thoma, Eur. Phys. J. A 24, 111 (2005)

[4] A. V. Anisovich and A. V. Sarantsev, Eur. Phys. J. A 30, 427 (2006)

[5] A. V. Anisovich, R. Beck, E. Klempt, V. A. Nikonov, A. V. Sarantsev and U. Thoma, Eur. Phys. J. A 48, 15 (2012) doi:10.1140/epja/i2012-12015-8 [arXiv:1112.4937 [hep$\mathrm{ph}]]$

[6] G. Agakishiev et al. [HADES Collaboration], Eur. Phys. J. A 51, no. 10, 137 (2015) doi:10.1140/epja/i2015-15137-5 [arXiv:1703.08398 [nucl-ex]]

[7] V. L. Kashevarov et al. [A2 Collaboration], JPS Conf. Proc. 13, 020029 (2017) doi:10.7566/JPSCP.13.020029 [arXiv:1702.06060 [nucl-ex]]

[8] M. Williams et al. [CLAS Collaboration], Phys. Rev. C 80, 045213 (2009) doi:10.1103/PhysRevC.80.045213 [arXiv:0909.0616 [nucl-ex]]

[9] N. Compton et al. [CLAS Collaboration], Phys. Rev. C 96, no. 6, 065201 (2017) doi:10.1103/PhysRevC.96.065201 [arXiv:1706.04748 [nucl-ex]]

[10] S.A. Pereira et al. [CLAS Collaboration], Phys. Lett. B 688, 289 (2010)

[11] S. Prakhov et al., Phys. Rev. C 80, 025204 (2009)

[12] D. F. Baxter et al., Nucl. Phys. B 67, 125 (1973)

[13] A. de Bellefon et al., Nuovo Cim. A 90, 1 (1975)

[14] J. Griselin et al., Nucl. Phys. B 93, 189 (1975)

[15] B. Conforto et al. [Rutherford-London Collaboration], Nucl. Phys. B 105, 189 (1976)

[16] W. Cameron et al. [Rutherford-London Collaboration], Nucl. Phys. B 193, 21 (1981)

[17] M. Jones, R. Levi Setti, D. Merrill and R. D. Tripp, Nucl. Phys. B 90, 349 (1975)

[18] R. Armenteros et al., Nucl. Phys. B 21, 15 (1970)

[19] A. Berthon et al., Nucl. Phys. B 20, 476 (1970)

[20] G. W. London et al., Nucl. Phys. B 85, 289 (1975) 